\title{
A Comparison of Amplitude-Phase Coupling and Linewidth Enhancement in Semiconductor Quantum-Well and Bulk Lasers
}

\author{
B. Zhao, T. R. Chen, and A. Yariv
}

\begin{abstract}
The amplitude-phase coupling factor $\alpha$ (linewidth enhancement factor) is compared in typical semiconductor quantum-well and bulk double heterostructure lasers. As a direct consequence of the reduction of the differential gain, there is no reduction of $\alpha$ in the single quantum-well lasers compared to the bulk lasers. The number of quantum wells strongly affects the amplitude-phase coupling in quantum-well lasers. It is shown that the interband transition induced amplitude-phase coupling still dominates over that induced by the plasma effect of carriers in the typical quantum-well lasers. By considering the spontaneous emission factor in the spectral linewidth we show that there is an optimal quantum-well number for achieving the narrowest spectral linewidth.
\end{abstract}

\section{INTRODUCTION}

$\mathrm{N}$ semiconductor lasers there is a fundamental linewidth Lenhancement resulting from coupling between spontaneous emission induced amplitude and phase perturbation. This leads to the modified Schawlow-Townes formula for the spectral linewidth of semiconductor lasers [1], [2];

$$
\Delta \nu=\frac{v_{g}^{2} h \nu g \alpha_{m} n_{s p}\left(1+\alpha^{2}\right)}{8 \pi P}
$$

where $v_{g}$ is the group velocity of the light, $h \nu$ is the lasing energy, $g$ is the modal gain, $\alpha_{m}$ is the mirror loss, $P$ is the optical output power, $n_{s p}$ is the spontaneous emission factor, and $\alpha$ is the linewidth enhancement factor which reflects the amplitude-phase coupling in the lasers. The amplitude-phase coupling factor $\alpha$ is of fundamental importance in semiconductor lasers. It plays a crucial role in determining the field linewidth, the chirping characteristics, and in any attempts to control these characteristics.

The reduction of the spectral linewidth of semiconductor lasers is important in optical communication, since a narrower spectral linewidth enables a larger number of communication channels by reducing the pulse spreading in digital systems and in analog systems, which are all influenced by signal distortion due to fiber dispersion. The

Manuscript received June 5, 1992. This work was supported by DARPA, the Office of Naval Research, and the NSF.

The authors are with T. J. Watson Sr. Laboratories of Applied Physics, California Institute of Technology, Pasadena, CA 91125.

IEEE Log Number 9207492. amplitude to phase coupling is also the major factor causing undesired frequency modulation (FM) (chirping) associated with the amplitude modulation (AM) [3] in semiconductor lasers. And in some circumstances the amplitude noise (or the phase noise) can be reduced through the decorrelation of phase-amplitude coupling [4] in semiconductor lasers.

It has been believed that quantum-well ( $Q W$ ) lasers would be a good candidate to achieve a much narrow spectral linewidth due to the reduction of linewidth enhancement factor $\alpha$ compared to that of bulk lasers [5], [6]. The single quantum-well (SQW) lasers were expected to be superior to their multiple quantum-well (MQW) counterparts in the spectral linewidth due to the smaller spontaneous emission factor $n_{s p}$ in SQW lasers [7]. The reduction of the $\alpha$ factor in the QW lasers has been attributed to the differential gain enhancement compared to bulk lasers [5], [6]. However, in our recent study, we found that there is a significant modification to the expected differential gain enhancement in QW lasers if the unavoidable thermal population of injected carriers in the optical confining region (state filling effect) is included [8], [9]. In this paper, we present an analysis which shows how the amplitude-phase coupling factor $\alpha$ and the spectral linewidth in QW lasers are influenced by the modification of the differential gain enhancement due to state filling.

The linewidth enhancement factor $\alpha$ is defined as

$$
\begin{aligned}
\alpha & =\left(\partial \chi_{r} / \partial N\right) /\left(\partial \chi_{i} / \partial N\right) \\
\chi(N) & =\chi_{r}+i \chi_{i}
\end{aligned}
$$

where $N$ is the injected carrier density, $\chi_{r}$ and $\chi_{i}$ are real and imaginary part of the effective optical susceptibility $\chi$ which are given by

$$
\begin{aligned}
\chi_{i}(E, N)= & -\Gamma \sum_{i=l, h} \int A_{i}(\mathcal{E}) \rho_{i, r}(\mathcal{E})\left(f_{e}+f_{h}-1\right) \\
& \cdot \mathcal{L}_{i}(\mathcal{E}-E) d \mathcal{E} \\
\chi_{r}(E, N)= & -\Gamma \sum_{i=1 . h} \int A_{i}(\mathcal{E}) \rho_{i, r}(\mathcal{E})\left(f_{e}+f_{h}-1\right) \\
& \cdot \mathcal{L}_{r}(\mathcal{E}-E) d \mathcal{E}
\end{aligned}
$$


where

$$
\mathcal{L}_{i}(\mathcal{E}-E)=\frac{\hbar / T_{2}}{\left(\hbar / T_{2}\right)^{2}+(\mathcal{E}-E)^{2}}
$$

and

$$
\mathcal{L}_{r}(\mathcal{E}-E)=\frac{\mathcal{E}-E}{\left(\hbar / T_{2}\right)^{2}+(\mathcal{E}-E)^{2}}
$$

$E$ is the photon energy, $T_{2}$ is the collisional dephasing time, $f_{e}$ and $f_{h}$ are the quasi-Fermi distribution functions for electrons and holes, respectively, $\rho_{i, r}$ is the density of states function which represents either the step-like twodimensional (2-D) reduced density of states for the QW structures or the parabolic three-dimensional (3-D) density of states for the bulk double heterostructure (DH), $i$ designates either light holes $(j=l)$ or heavy holes $(j=$ $h), A_{i}(\mathcal{E})$ includes the transition dipole moment and the polarization modification factor for the dipole moment in the QW structure. $\Gamma$ is the conventional confinement factor for the DH structures and is equal to $1 / t$ for the QW structures, where $t$ is the effective lateral width of the optical mode. The modal gain of the laser devices is related to $\chi_{i}$ by

$$
g(E, N)=-\frac{E}{c \hbar n_{r}} \chi_{i}(E, N)
$$

where $n_{r}$ is the modal effective refractive index.

We calculated the $\alpha$ parameter at the modal gain peak for typical GaAs-AlGaAs QW and DH lasers. For QW structure we assume a symmetric $4000 \AA \mathrm{Al}_{0.5} \mathrm{Ga}_{0.5} \mathrm{As}$ $\mathrm{Al}_{0.25} \mathrm{Ga}_{0.75} \mathrm{As}$ graded index separate confinement heterostructure (GRINSCH) with $100 \AA \mathrm{GaAs}$ quantum wells at its center. For DH lasers we assume an $\mathrm{Al}_{0.35} \mathrm{Ga}_{0.65} \mathrm{As}-$ $\mathrm{GaAs} \mathrm{DH}$ with $\mathrm{GaAs}$ active layer thickness $0.1 \mu \mathrm{m}$. The calculation is made for the dominant $\mathrm{TE}$ mode in the DH lasers and in the GRINSCH SQW, three quantum wells $(3 \mathrm{QW})$, and five quantum wells (5QW) lasers, respectively. We have assumed parabolic and uncoupled subbands. Typical values of effective masses for electrons and holes in GaAs material are adopted and $T_{2}=0.1 \mathrm{ps}$ is used.

In Fig. 1 we show the $\alpha$ parameter at the gain peak as a function of the modal gain. It is important to note that there is no reduction of $\alpha$ parameter in the SQW structure. On the contrary, the $\alpha$ parameter in SQW structure is much larger than that in DH structure. The explanation to this is the following. A decrease of carrier density (due to spontaneous emission) results in a decrease of quasi-Fermi energies. The change of Fermi energies is relatively small due to the unavoidable thermal population of carriers among the large density of states in the SCH confining region. The carrier density change in the $\mathrm{SCH}$ confining region contributes relatively little to the change of $\chi_{i}$ due to the nonresonance nature of $\mathcal{L}_{i}(\mathcal{E}-E)$ [see (6)] and the flat feature of the 2-D step-like density of states in the

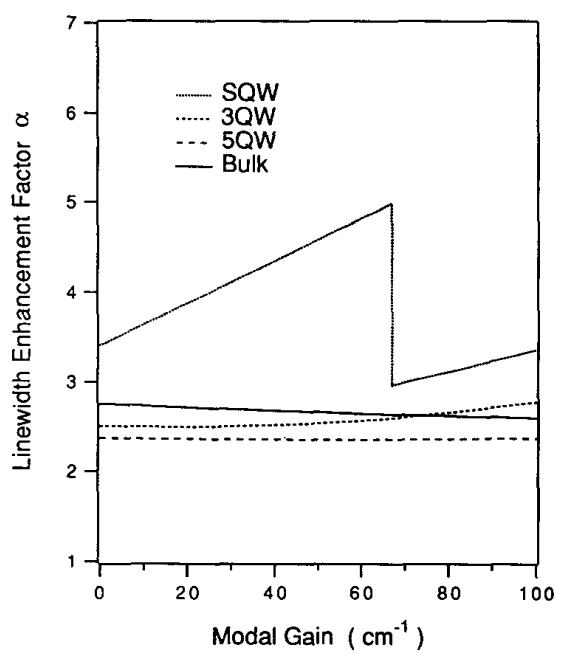

Fig. 1. The linewidth enhancement factor $\alpha$ (interband transition component) at optical gain peak as a function of the modal gain in the bulk DH lasers and the $\mathrm{QW}$ lasers with different number of quantum wells.

QW. The resulting change in the denominator of (2) is thus small. On the other hand, the carrier density change in $\mathrm{SCH}$ confining region contributes a relatively large amount to the change of $\chi_{r}$ due to the long tail of $\mathscr{L}_{r}(\mathcal{E}$ $-E$ ) at high energy [see (7)]. Apparently, a large carrier population in the $\mathrm{SCH}$ region will enhance this effect. This leads to an increase of the $\alpha$ parameter in the SQW lasers. The abrupt drop of the $\alpha$ curve for the SQW structure in Fig. 1 is due to the onset of the second quantized state lasing which leads to a differential gain [or the denominator in (2)] enhancement. Fig. 1 also shows that the use of MQW leads to a reduction $\alpha$. This is attributed to the reduction of the state filling in the $\mathrm{SCH}$ optical confining region [9], which results in an additional differential gain enhancement in MQW lasers. However, the reduction of $\alpha$ in the MQW structures is not significant in comparison to the $\alpha$ in bulk DH structures.

Generally the amplitude and phase coupling in semiconductor lasers consists of an interband transition component (as we have discussed above and shown in Fig. 1) and a free-carrier component. The free-carrier component of $\alpha$ stems from the plasma effect of injected carriers and has been shown to be much smaller than the interband transition component in bulk lasers [10]. We plotted in Fig. 2 the calculated free-carrier component of $\alpha$ at the optical gain peak in the assumed QW and bulk lasers as a function of the modal gain. It was suggested that the carrier fluctuation in the optical confining layers affect the amplitude-phase coupling through a significantly large free-carrier component of $\alpha$ in SCH QW lasers [11]. We show in Fig. 2, comparing with Fig. 1, that the interband transition component of $\alpha$ is still dominant in the QW lasers. Fig. 2 shows that the free-carrier component of $\alpha$ is larger in SQW lasers and is smaller in MQW lasers compared to the bulk lasers. The explanation is referred to the differential gain behavior in these lasers. 


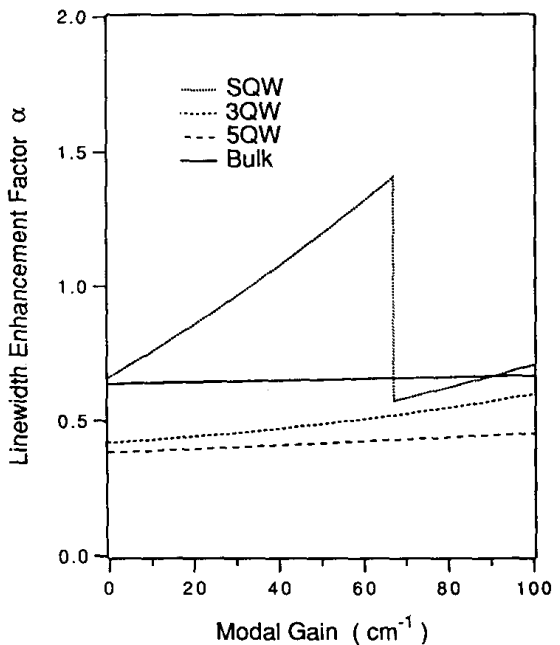

Fig. 2. The linewidth enhancement factor $\alpha$ (free-carrier component) at optical gain peak as a function of the modal gain in the bulk DH lasers and the QW lasers with different number of quantum wells.

Equation (1) shows that, in addition to the $\alpha$ parameter, the spontaneous emission factor $n_{s p}$ also plays an important role in determining the spectral linewidth of semiconductor lasers. $n_{s p}$ is the ratio of the spontaneous emission rate into the lasing mode to the stimulated emission rate and is given by

$$
\begin{aligned}
n_{s p}(E, N) & \\
& =\frac{\sum_{i=l, h} \int A_{i}(\mathcal{E}) \rho_{i, r}(\mathcal{E}) f_{e} f_{h} \mathcal{L}_{i}(\mathcal{E}-E) d \mathcal{E}}{\sum_{i=l, h} \int A_{i}(\mathcal{E}) \rho_{i, r}(\mathcal{E})\left(f_{e}+f_{h}-1\right) \mathcal{L}_{i}(\mathcal{E}-E) d \mathcal{E}} .
\end{aligned}
$$

The calculated spontaneous emission factor at the gain peak as a function of the modal gain for the assumed QW and bulk DH lasers is plotted in Fig. 3. It shows that QW lasers possess lower $n_{s p}$ than that of DH lasers. The SQW lasers have the smallest $n_{s p}$. This is due to the 2-D flat density of states distribution in the QW structures, which makes it necessary to have a larger inversion in QW structures compared to bulk DH structures in order to achieve the same modal gain.

Equation (1) shows that $n_{s p}\left(1+\alpha^{2}\right)$ is the figure of merit for the spectral linewidth in semiconductor lasers of different structures. Notice that $\alpha$ decreases and $n_{s p}$ increases in the QW lasers as the number of quantum wells increases, there might be an optimal quantum-well number for achieving the narrowest spectral linewidth. To compare the linewidth enhancement in different laser structures, we plot $n_{s p}\left(1+\alpha^{2}\right)$ at the gain peak as a function of the modal gain in Fig. 4, where both the interband transition component and the free-carrier component of $\alpha$ are included. It can be seen that MQW lasers possess narrower linewidth compared to bulk lasers and this is attributed mainly to the smaller $n_{s p}$ in the MQW lasers. The linewidth of the SQW lasers can be either smaller or larger than that of the bulk lasers depending on the modal gain.

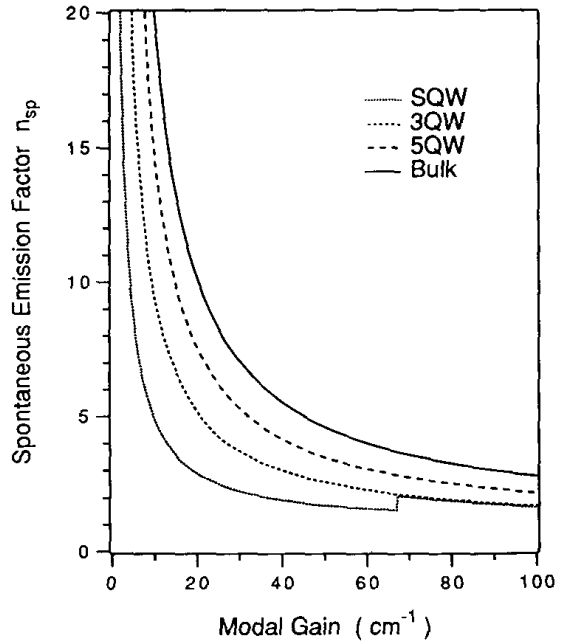

Fig. 3. The spontaneous emission factor $n_{s p}$ at optical gain peak as a function of the modal gain in the bulk DH lasers and the QW lasers with different number of quantum wells.

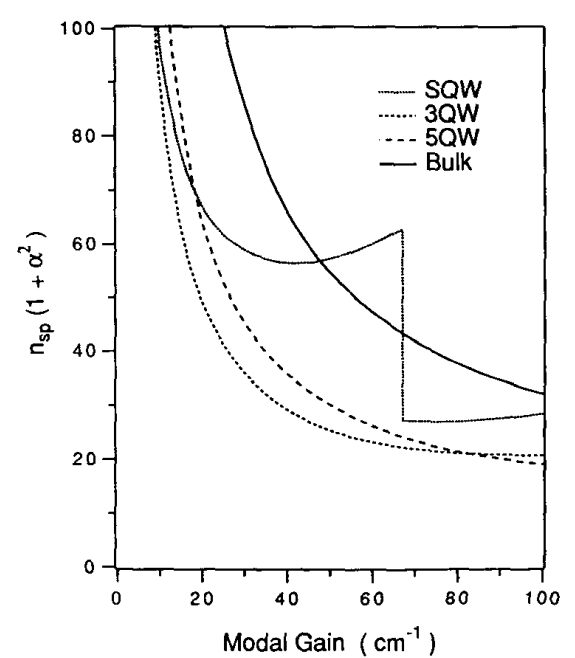

Fig. 4. The figure of merit for the spectral linewidth enhancement in semiconductor lasers $n_{s p}\left(1+\alpha^{2}\right)$ at optical gain peak as a function of the modal gain in the bulk $\mathrm{DH}$ lasers and the QW lasers with different number of quantum wells.

\section{CONCLUSION}

In conclusion, we find that there is an enhancement in the amplitude-phase coupling in SQW lasers compared with that of bulk DH lasers. This is due mainly to the reduction in differential gain caused by the state filling effect in SQW lasers. The amplitude-phase coupling is reduced in MQW lasers due to the extra differential gain enhancement. It is shown that the interband transition induced amplitude-phase coupling is dominant compared to that induced by the plasma effect of injected carriers in typical QW lasers. Compared to bulk lasers, the reduction of amplitude-phase coupling is not significant in MQW lasers. Due to the smaller spontaneous emission factor $n_{s p}$ 
in QW lasers, the QW lasers may have narrower linewidth than that of the bulk lasers and there is an optimal QW well number for achieving the narrowest spectral linewidth in the QW lasers.

\section{REFERENCE}

[1] C. H. Henry, "Theory of the linewidth of semiconductor lasers," IEEE J. Quantum Electron, vol. QE-18, pp. 259-264, 1982.

[2] K. Vahala, L. C. Chiu, S. Margalit, and A. Yariv, "On the linewidth enhancement factor $\alpha$ in semiconductor injection lasers," Appl. Phys. Lett., vol. 42, pp. 631-633, 1983.

[3] C. Harder, K. Vahala, and A. Yariv, "Measurement of the linewidth enhancement factor $\alpha$ of semiconductor lasers," Appl. Phys. Lett., vol. 42 , pp. $328-330,1983$

[4] K. Vahala and M. Newkirk, "Intensity noise reduction in semiconductor lasers by amplitude-phase decorrelation," Appl. Phys. Lett., vol. 57, pp. 974-976, 1990
[5] M. G. Burt, "Linewidth enhancement factor for quantum-well lasers," Electron. Lett., vol. 20, pp. 27-29, 1984.

[6] Y. Arakawa and A. Yariv, "Quantum noise and dynamics in quantum well and quantum wire lasers," Appl. Phys. Lett., vol. 45, pp. 950$952,1984$.

[7] - "Theory of gain, modulation response, and spectral linewidth in AlGaAs quantum well lasers,"' IEEE J. Quantum Electron., vol. QE-21, pp. 1666-1674, 1985 .

[8] B. Zhao, T. R. Chen, and A. Yariv, "Effect of state-filling on the modulation response and the threshold current of quantum well lasers," Appl. Phys. Lett., vol. 60, pp. 1930-1932, 1992.

[9] - "The extra differential gain enhancement in multiple quantum well lasers," IEEE Photon. Technol. Lett., vol. 4, pp. 124-126, 1992.

[10] C. H. Henry, R. A. Logan, and K. A. Bertness, "Spectral dependence of the change in refractive index due to carrier injection in $\mathrm{GaAs}$ lasers," J. Appl. Phys., vol. 52, pp. 4457-4461, 1981.

[11] M. Yamada and Y. Haraguchi, "Linewidth broadening of SCH quantum well lasers enhanced by carrier fluctuation in optical guiding layers," IEEE J. Quantum Electron., vol. 27, pp. 1676-1681, 1991. 